Pierre-Alain Duc, Jonathan Braine and Elias Brinks, eds.

\title{
Some Effects of Galaxy Collisions in a Cluster ICM
}

\author{
Curtis Struck, Jason R. Brown \\ Dept. of Physics \& Astronomy, Iowa State Univ., Ames, IA 50011, USA
}

\begin{abstract}
We present preliminary results of numerical modeling of the combined effects of ICM stripping and galaxy collisions on galaxies in clusters. We use the Hydra adaptive mesh, P3M + SPH code of Couchman et al. (1995). Generally, the formation of extended tidal and splash structures in galaxy interactions will facilitate ram pressure stripping. ICM compression of massive clouds stripped from tails and plumes may produce significant populations of free-floating star clusters or dwarf galaxies.
\end{abstract}

There has been suggestions in the literature that galaxy collisions are more common in the environment of galaxy clusters (e.g., Fried 1988, Rubin, Waterman \& Kenney 1999). The cumulative effects of long range tidal disturbances, i.e., galaxy harrassment, can produce some similar results (see Moore et al. 1996), and so complicates the interpretation of observation. However, secular processes are unlikely to have had time to affect gas-rich galaxies in groups or subclusters falling into large clusters. In this case cluster potential may induce interactions.

There are some beautiful candidate examples of galaxy collisions in clusters. These include: 1) NGC 4438/35 = Arp 120 studied by Kenney et al. (1995, and Kenney \& Yale, 2002). These authors also find evidence of an AGN or starburst driven outflow. 2) NGC 4485/90 studied by Clemens et al. (2000, with refs. to earlier papers). Following the encounter, the smaller galaxy may have had all of its gas stripped. 3) NGC 4388 studied by Yoshida et al. (2002, and poster at this meeting), which has long, extra-disk, emission line spirals like those in the models below.

We would expect that the gas in tidal tails and splash bridges or plumes would be more easy to strip than gas in disks, and because of this galaxy collisions would enhance ram pressure stripping. We also expect ICM compression to facilitate star formation in gas clouds contained within tidal structures, while ram pressure may push some of these clouds free of their parent galaxies.

To date there has been little modeling work undertaken to study these possibilities. We are beginning work in this area, and present preliminary results here. Specifically, we initialize a galaxy consisting of an isothermal, spherically symmetric dark matter halo, plus disk consisting of half star and half gas particles distributed symmetrically around the $\mathrm{x}-\mathrm{y}$ plane, The galaxy is impacted by an hot ICM 'wind' coming from the positive z-direction, and travelling parallel to the $\mathrm{z}$-axis with a velocity of $2000 \mathrm{~km} \mathrm{~s}^{-1}$ relative to the galaxy disk, as in the models of Schulz \& Struck (2001). In addition, we define a circular region within the disk, centered at half the disk radius $(\mathrm{x}=0.5, \mathrm{y}=0.5$ in model units), with its radius equal to about one third of the galaxy disk radius. Within that circle 
the gas particles are given a negative velocity in the $\mathrm{z}$ direction of $300 \mathrm{~km} \mathrm{~s}^{-1}$, as though the disk had been directly impacted by a smaller disk.

The impacted gas is pushed down, but not immediately, nor entirely stripped (see Figure 1). Eventually, we find that stripping is enhanced by this collisional disturbance - about $30 \%$ of the disk gas is removed, versus $18 \%$ in the corresponding non-collisional case. This is true even without the formation of extended tidal tails, which should be easy to strip. The remnant disk is much more disturbed in the collisional case.

As in stripping calculations without a collision, ram pressure stimulates the formation of spiral arms in the gas disk (via the annealing process of Schulz and Struck 2001). The outer parts of these arms are stripped and compressed, with the formation of clumps in the process. These clumps are good candidate sites for the formation of young star clusters or dwarf galaxies. We would expect the same phenomena in tidal tails.

Angular momentum transfer is more vigorous in the collision than in the comparison run. As a result, gas in the remnant disk core is more compressed and would likely be the site of a subsequent starburst.
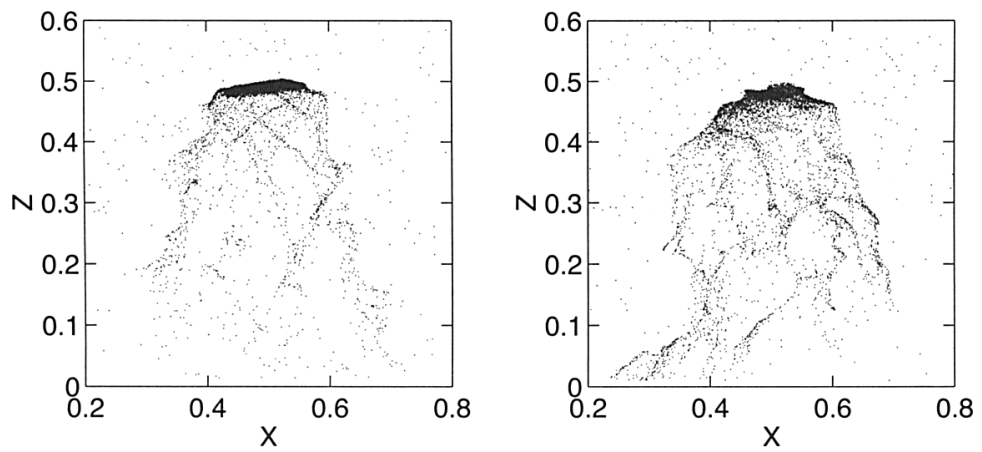

Figure 1. Edge-on views of gas particles in a galaxy disk moving face-on into an ICM wind at a time of 94 Myr after the onset of the wind. The grid scale is $100 \mathrm{kpc}$. The model in the right panel has collided with a smaller disk as described in the text.

\section{References}

Clemens. M., Alexander, P., \& Green, D. A. 2000, MNRAS, 312, 236

Couchman, H. M. P., Thomas, P. A., \& Pearce, F. R. 1995, ApJ, 452, 797

Fried, J. W. 1988, A\&A, 189, 42,

Kenney, J. P. D., Rubin, V. C., Planesas, P., \& Young, J. S. 1995, ApJ, 438, 135

Kenney, J. P. D., \& Yale, E. E. 2002, ApJ, 567, 865

Moore, B., et al. 1996, Nature, 379, 613

Rubin, V. C., Waterman, A. H., \& Kenney, J. P. D. 1999, AJ, 118, 236

Schulz, S., \& Struck, C. 2001, MNRAS, 328, 185

Yoshida, M., et al. 2002, ApJ, 567, 118 